\title{
El impacto de las remesas en la pobreza de ingresos de los hogares salvadoreños
}

Álvaro Castro

\section{Introducción}

El pensamiento económico predominante asegura, en cierta forma, que la globalización llevará a una amplia mejoría de los ingresos promedio; que habrá más y mejores economías de escala, a medida se encuentren más mercados, que los ingresos convergerán a medida que los países pobres crezcan relativamente más rápido que los ricos. Es la llamada "aldea global", donde la integración de mercados y la prosperidad surgen. Pero la realidad es obviamente distinta: la brecha entre los países ricos y pobres se ha ensanchado, así como la desigualdad interna entre estos últimos. Asimismo, la libre movilidad de mercados no se cumple: los mercados de bienes y de cápital financiero están muy abiertos, pero las naciones industrializadas tienen fuertes barreras contra la inmigración, haciendo de la movilidad humana algo generalmente asociado con inmigración ilegal. A través del tiempo, la migración de cápital humano ha tenido diversos efectos en las sociedades afectadas por ella. Actualmente, por ejemplo, el masivo movimiento migratorio de latinoamericanos a EE.UU., ha llevado de manera relativamente rápida, a que se conviertan en el grupo étnico más grande de tal nación. ${ }^{\prime}$ 
En general, el movimiento de inmigrantes latinos que buscan una mejoría en su calidad de vida, trae el surgimiento de ayuda enviada por ellos hacia sus lugares de origen. Esta ayuda, ya sea en dinero y/o en especie, llamada remesa, la cual, en el caso de muchos países latinoamericanos constituye un constante flujo de ayuda unilateral hacía sus habitantes. Usualmente son los de menores ingresos, a quienes su sostenimiento económico se les haría más difícil si disminuyeran o faltaran por completo. El Salvador es un buen representante de tal fenómeno: a nivel macroeconómico las cifras mostradas por el Estado muestran cómo las transferencias en concepto de remesas llegaron a constituir hasta un $14 \%$ del Producto Interno Bruto en la pasada década, aminorando en cierta medida el impacto de factores negativos de la economía como el creciente déficit de cuenta corriente. A nivel microeconómico, se conoce popularmente que las remesas ayudan en gran parte al sustento del hogar. Alrededor de esta reflexión surgen creencias sobre las diferencias que los hogares beneficiados puedan tener con aquellos que no reciben ayuda alguna, como el número de miembros que efectivamente laboran, los montos recibidos y el subsiguiente bienestar, etc.

El siguiente artículo sintetiza los resultados encontrados en la investigación del mismo nombre $e^{2}$ en donde se buscó en primer lugar, determinar el impacto de la remesas en el nivel de bienestar de los hogares salvadoreños, así como la proporción que éstas representan en los ingresos de los hogares, las diferencias socioeconómicas entre los hogares receptores y los que no obtienen ingresos de este tipo, además, se compara la situación de pobreza de los salvadoreños con el contexto hipotético de no recibir remesa alguna. Los resultados despejaron las dudas que se tenían sobre las remesas y sus receptores, y confirmaron categóricamente el fuerte papel que juegan estas en los hogares salvadoreños, que al fin y al cabo, son los elementos que forman a la sociedad y que en conjunto forma la nación, además de inferir algunas conclusiones con respecto a los resultados de la política social del actual gobierno.

\subsection{Condición de pobreza en El Salvador}

Al observar cifras del Banco Mundial, es evidente el bajo lugar que ocupa El Salvador en la clasificación de ingreso, El Salvador es calificado como de ingreso medio bajo ${ }^{3}$. Estas particularidades muestran 
cómo, al compararse las tendencias de los demás países con niveles de ingreso similares, El Salvador presenta promedios de inversión menores y ahorro doméstico ínfimo. Esto, al combinarse con otros factores como la creciente desigualdad en la distribución del ingreso, que según el Informe de Desarrollo Humano 2002, es una de las más altas del mundo (el $20 \%$ más rico de la población percibe, en promedio, ingresos 18 veces más altos que el $20 \%$ de los más pobres), permite inferir la existencia de pobreza en el país.

Las variables sociodemográficas y económicas publicadas por el Banco Mundial en 2002, muestran a un país con pobres expectativas de vida, de crecimiento y de desarrollo para sus generaciones más jóvenes: la desnutrición infantil en menores de cinco años es de $12 \%$ (mayor al promedio del resto de América Latina y el Caribe por tres puntos porcentuales); la mortalidad infantil es de $29 \%$; el analfabetismo en mayores de 15 años es del $21 \%$. Adicionalmente, las expectativas de crecimiento del PIB per cápita en el quinquenio de 2001-2005 sean de apenas un $1.8 \%$.

\subsection{Ingreso y pobreza en los hogares salvadoreños}

De los 6,510,348 personas concentradas en los 1,522,383 hogares existentes hasta 2002 , el $59.0 \%$ de hogares se en encuentran ubicados en el área urbana y el $\mathbf{4 1 . 0 \%}$ en el área rural. Además, la tasa de variación poblacional de los últimos cuatro años es de $5.8 \%$, lo que indica que lejos de estacionalizarse o disminuir, la población salvadoreña va en marcado aumento.

\section{Gráfico No 1}

Número de hogares desde 1999 hasta el 2002

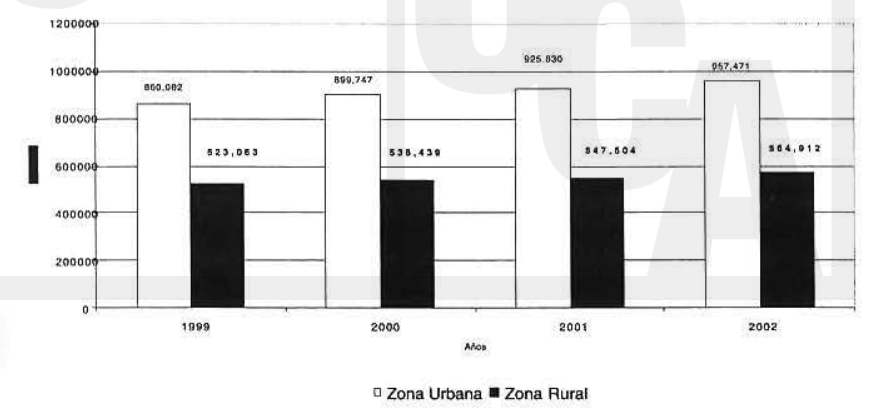

Fuente: Elaboración propia en base a datos de la Encuesta de Hogares de Propósitos Múltiples (EHPM). 
Además, la cantidad de miembros por hogar es, en promedio, de 4.6 (4.2 en la zona urbana; 4.9 en la zona rural), lo que indica un buen número de personas que viven bajo un mismo techo. Los departamentos que tienen mayor número de miembros promedio por unidad familiar son, Cabañas, Sonsonate y Ahuachapán. En contraste, los que presentan un promedio menor de personas que integran sus respectivos hogares, son San Miguel, Santa Ana, San Salvador y Usulután. Los hogares clasificados urbanos y que tienen los indicadores más altos según la investigación, son: Cuscatlán, Cabañas y San Vicente; y en la zona rural son Cabañas y Sonsonate; mientras que los hogares con el menor promedio de miembros son los de Santa Ana, y San Miguel en la zona urbana; San Miguel, Usulután y San Salvador para el área rural.

\subsection{Ingreso familiar: su evolución y distribución}

El ingreso familiar nominal promedio fluctuó hacia el alza en el período estudiado, aunque como país integrado a la economía globalizada, El Salvador ha resentido los efectos de la recesión mundial, además de su propia, débil coyuntura. Esta de más señalar que la zona rural es la que tiene los menores ingresos promedio. Ahora bien, en la zona urbana, los departamentos que mayor ingreso promedio presentan, son La Libertad (\$726 mensuales) y San Salvador (\$556). Por el contrario, los departamentos que presentan menores ingresos, son Cabañas (\$411 mensuales), Ahuachapán (\$336) y Usulután (\$432). En lo rural, los departamentos que mayores ingresos presentan, son La Libertad (\$295 mensuales), Cuscatlán (\$277) y San Salvador (\$266). Los que tienen menores ingresos, son Cabañas, San Vicente (ambos con $\$ 171$ mensuales) y Usulután (\$193). Los departamentos con mayor ingreso promedio, citados anteriormente, albergan a gran parte de habitantes con altos ingresos. Por esto, se explica la superioridad de ingreso. 


\section{Gráfico $\mathrm{N}^{\circ} 2.1$ \\ Rango de Ingreso Familiar Mensual Urbano \\ (En Dólares)}

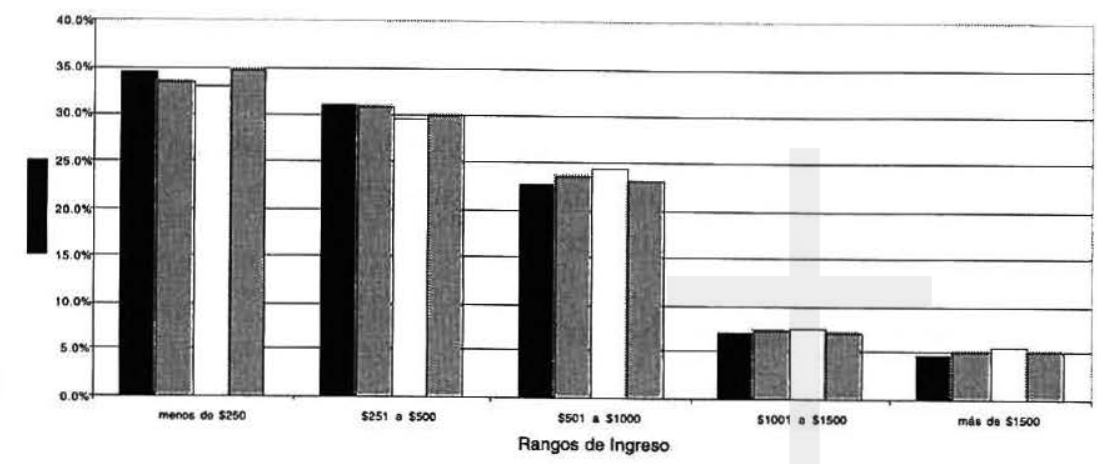

- AÑO 1995 AÑO $2000^{\circ}$ AÑo $200 \%$ AÑO 2002

Fuente: Elaboración propia en base a datos de la EHPM.

\section{Gráfico $\mathrm{N}^{\circ} 2.2$}

\section{Rangos de Ingreso Familiar Mensual Rural}

(En Dólares)

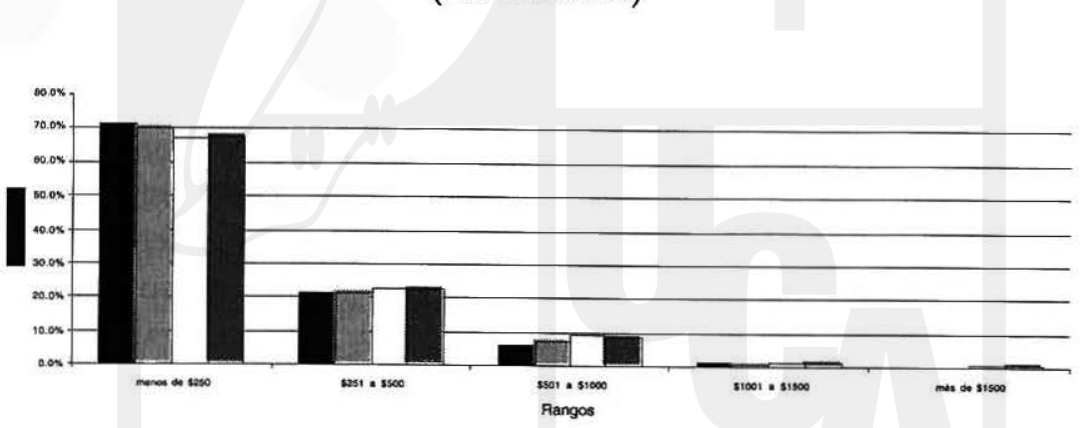

- AÑO 1999٪ AÑO 2000 - AÑO 2001٪ AÑO 2002

Fuente: Elaboración propia en base a datos de la EHPM.

La distribución del ingreso entre ambas zonas es claramente desigual. Al analizar las gráficas, se ve como a lo largo de los cuatro años, 
el porcentaje de hogares urbanos presenta una mejor en comparación con sus homólogos rurales: aproximadamente un $85 \%$ de los ingresos urbanos se distribuyen en los rangos de ingreso de menos de $\$ 250$ a $\$ 1000$, mientras que en la zona rural ese mismo porcentaje de la población, se encuentra en los rangos de ingresos que corresponden a ingresos hasta $\$ 500$.

El análisis demostró que existió en el período estudiado un leve crecimiento en el ingreso de los hogares salvadoreños, en lo general. Este aumento no produjo una mejoría sustancial de las condiciones socio-económicas de los habitantes.

\subsection{Ingreso per cápita por hogar}

El país presenta bajos ingresos per cápita: en promedio, este sólo ha sido de $\$ 90$ mensual en los últimos cuatro años; si se divide el análisis por zonas se confirma lo dicho anteriormente sobre la desigualdad en poder adquisitivo de las personas: un habitante urbano tiene como ingreso promedio, $\$ 127$ al mes; mientras que uno rural sólo $\$ 53$, una diferencia del $59 \%$ aproximadamente. La Libertad y San Salvador sobresalen como los departamentos que poseen ingresos per cápita mensuales mas elevados a nivel nacional, con montos promedio de $\$ 212$ y $\$ 169$ respectivamente, en la zona urbana; y asimismo, con $\$ 69$ y $\$ 64$ en la zona rural.

Si comparamos el ingreso mensual per cápita del departamento que recibe mayores ingresos con el que se encuentra en la situación opuesta, podemos observar que el ingreso per cápita promedio en el departamento de La Libertad es $93.3 \%$ mayor que el ingreso por persona en Cabañas; en la zona urbana, este porcentaje se eleva y es de $117.4 \%$. En la zona rural, La Libertad posee un ingreso per cápita promedio de $95.8 \%$, mayor que el ingreso de la zona rural del departamento de Cabañas.

En cuanto a las tasas de variación que ha presentado el ingreso per cápita salvadoreño en los años en estudio, este muestra como, a excepción de Ahuachapán, ha ido hacia el alza, creciendo un $16.2 \%$ en el área rural y un $9.2 \%$ en la urbana. Posteriormente se comprueba que este aumento se debe a las remesas y no por la eficiencia del aparato productivo, ni por una mejoría en la distribución del ingreso. 
Cuadro $\mathrm{N}^{\circ} 1$

Ingreso Per cápita 1999- 2002

\begin{tabular}{|c|c|c|c|c|c|}
\hline \multirow[t]{2}{*}{ Departamento } & \multicolumn{3}{|c|}{ Promedio } & \multirow{2}{*}{$\begin{array}{l}\text { Tasa } \\
\text { Crec. } \\
\text { Urbano }\end{array}$} & \multirow{2}{*}{$\begin{array}{l}\text { Tasa } \\
\text { Crec. } \\
\text { Rural }\end{array}$} \\
\hline & Urbano & Rural & País & & \\
\hline Ahuachapán & 97 & 48 & 73 & $-2.6 \%$ & $11.3 \%$ \\
\hline Santa Ana & 122 & 54 & 88 & $13.0 \%$ & $23.3 \%$ \\
\hline Sonsonate & 127 & 50 & 88 & $10.4 \%$ & $10.9 \%$ \\
\hline Chalatenango & 117 & 48 & 82 & $6.6 \%$ & $8.4 \%$ \\
\hline La Libertad & 212 & 69 & 140 & $6.1 \%$ & $17.8 \%$ \\
\hline San Salvador & 168 & 64 & 116 & $13.0 \%$ & $22.2 \%$ \\
\hline Cuscatlán & 117 & 67 & 92 & $1.2 \%$ & $6.2 \%$ \\
\hline La Paz & 112 & 57 & 84. & $6.1 \%$ & $15.5 \%$ \\
\hline Cabañas & 110 & 35 & 73 & $9.1 \%$ & $13.0 \%$ \\
\hline San Vicente & 113 & 41 & 77 & $7.2 \%$ & $13.6 \%$ \\
\hline Usulután & 124 & 49 & 86 & $19.0 \%$ & $22.8 \%$ \\
\hline San Miguel & 132 & 49 & 90 & $4.3 \%$ & $7.9 \%$ \\
\hline Morazán & 113 & 48 & 80 & $22.7 \%$ & $27.5 \%$ \\
\hline La Unión & 116 & 60 & 88 & $12.4 \%$ & $26.1 \%$ \\
\hline $\begin{array}{l}\text { Total Promedio } \\
\text { (En Dólares) }\end{array}$ & 127 & 53 & 90 & $9.2 \%$ & $16.2 \%$ \\
\hline
\end{tabular}

Fuente: Elaboración propia en base a datos de la EHPM.

\subsection{Pobreza extrema}

Aproximadamente $20 \%$ de los hogares de todo el país está situado debajo de la línea de la pobreza extrema. ${ }^{4}$ Los datos muestran que el porcentaje de pobres extremos ha disminuido en ambas zonas, nótese además que los pobres extremos rurales casi doblan a sus equivalentes urbanos y que el porcentaje los primeros no se ha reducido desde 2000. 


\section{Gráfico $\mathrm{N}^{\circ} 3$}

Porcentaje de hogares en pobreza extrema de 1999 a 2002

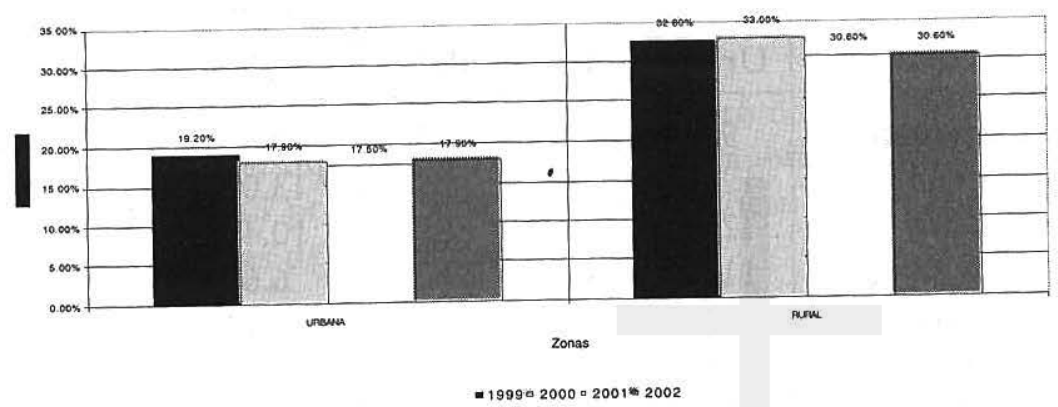

Fuente: Elaboración propia, sobre las bases de datos EHPM.

El ingreso promedio de los hogares situados debajo de la línea de la pobreza extrema, presentó una diferencia significativa entre el área rural y urbana. En lo urbano, el promedio es de \$76; y en lo rural es de $\$ 50$ al mes. Para ilustrar mejor la situación, los miembros de los hogares pobres urbanos que viven en pobreza extrema, sobreviven con $\$ 0.80$ diarios; los rurales, con $\$ 0.40$ diarios.

\section{Gráfico $\mathrm{N}^{\circ} 4$}

Ingreso familiar promedio de los hogares en pobreza extrema (En Dólares)

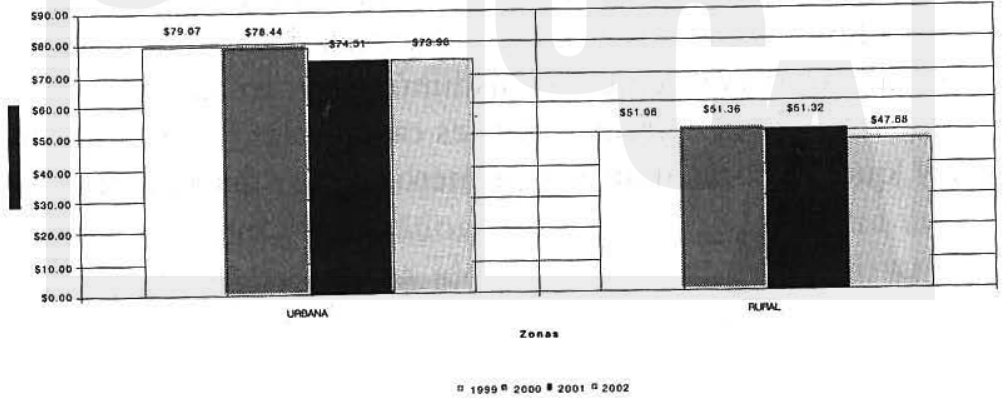

Fuente: Elaboración propia, sobre las bases de datos EHPM. 
La información por departamento muestra que en el área urbana, los departamentos que tienen menores ingresos per cápita mensuales son: Chalatenango con $\$ 18.58$ ( $\$ 0.62$ diarios) y Cabañas, con $\$ 19.02$ (\$0.63 diarios). Los que tienen más son La Libertad, con \$27.67 (\$0.92 diarios) y San Salvador, con $\$ 27.80$ ( $\$ 0.93$ diarios). En la zona rural, los más afectados a lo largo del período son Cabañas, con $\$ 9.01$ ( $\$ 0.30$ al día) y Ahuachapán, con $\$ 10.02$ al mes ( $\$ 0.33$ diarios).

Es penoso revelar que la cantidad de dinero con la que disponen los pobres extremos en las dos zonas, muestra la sumamente escasa capacidad adquisitiva de estos. Aún más, tal condición casi nulifica su acceso a los requerimientos mínimos de vivienda, alimentación y vestido.

La miseria en la zona rural es el doble que en la zona urbana, a tal grado de evidenciarse la extrema desigualdad existente entre ambas zonas.

\subsection{Pobreza relativa}

$\mathrm{Al}$ analizar la condición de pobreza relativa ${ }^{5}$ de los hogares, se encontró que aproximadamente el $43.9 \%$, de estos obtienen ingresos debajo de la canasta ampliada. Es importante destacar, que entre la zona urbana y rural, siguen habiendo diferencia significativas, ya que en lo urbano, el $40 \%$ de los hogares son pobres relativos; mientras que en la zona rural dicho porcentaje se eleva en un $20 \%$. Tal situación demuestra la gravedad y profundidad de la pobreza acentuada en la zona rural.

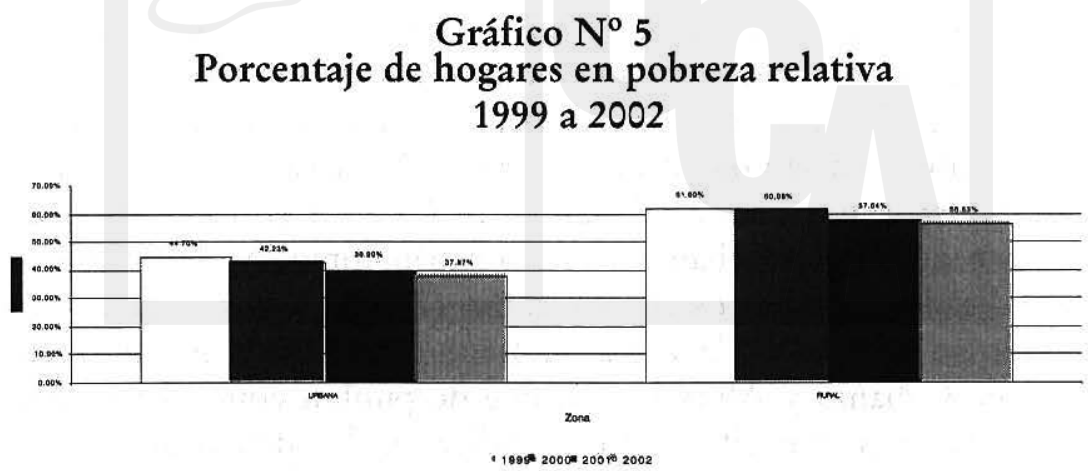

Fuente: Elaboración propia, sobre las bases de datos EHPM. 
La situación de desigualdad entre ambas zonas se ve también reflejada en el ingreso promedio de los hogares que caen por debajo de la canasta ampliada, debido a que en la zona urbana, el promedio fue igual a $\$ 143$ mensuales; en lo rural, dicho promedio fue de $\$ 93$.

\section{Gráfico $\mathrm{N}^{\circ} 6$}

Ingreso familiar promedio de los hogares en pobreza relativa (En Dólares)

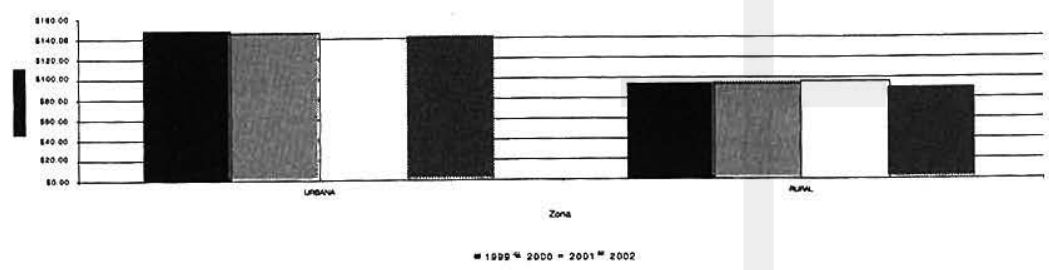

Fuente: Elaboración propia, sobre las bases de datos EHPM.

\subsection{Tasa media de dependencia}

La tasa media de dependencia nos muestra la proporción de individuos desocupados en relación a las personas ocupadas de sus hogares correspondientes; este indicador cuantifica el número de desocupados por cada ocupado por hogar, y es el resultado de la resta entre el número de personas por hogar menos el número de ocupados, todo dividido por el número de ocupados en dicho hogar.

En El Salvador, entre los años 1999 y 2002, la tasa de dependencia promedio para los hogares es de 2.0; 1.7 en la zona urbana y 2.2 en la zona rural, es decir, en promedio, en los hogares salvadoreños existen dos personas desocupadas por cada ocupado. En Cabañas, Ahuachapán, San Vicente y La Unión, la tasa de dependencia es mayor que la del resto de departamentos, oscilando entre 2.3 a 2.1 en promedio. En la zona urbana repiten Cabañas y Ahuachapán como los departamentos con indicadores más altos en este rubro ( 2.0 y 1.9 por hogar); y esto también se observa en la zona rural con 2.6 y 2.5 , respectivamente. Fuera de Cabañas y Ahuachapán, que despuntan como departamentos con las más altas cifras en este indicador, los demás departamentos son muy semejantes. La diferencia en la zona urbana entre Caba- 
ñas y el departamento que presenta un menor indicador por hogar es de 0.4; y para la zona rural es de 0.6 , y destacan para ambas zonas $\mathrm{La}$ Libertad y San Salvador con las tasas de dependencia más bajas (1.6 urbano, 2.0.rural). Cabe señalar que Cabañas y Ahuachapán son departamentos que presentan un gran número de hogares en situación de pobreza y por lo contrario, San Salvador y La Libertad son dos de los departamentos que obtienen mayores ingresos.

La tasa de dependencia se mantuvo estable en los años estudiados, reduciéndose tan sólo en un 0.1 en promedio tanto en el área urbana como en la rural. Los departamentos que presentan la reducción más significativa son Chalatenango y San Salvador, en el área urbana, con una reducción del 19.9 y 11.1 por ciento, respectivamente; en la zona rural, $\mathrm{La} \mathrm{Paz}$ y de nuevo, San Salvador destacan con las reducciones más significativas, en un 14.3 y 11.4 puntos porcentuales cada uno.

Lo que viene a significar una mayor tasa de dependencia para un hogar es una limitación de su capacidad de generar ingreso, y por lo tanto un impedimento a suplir las necesidades básicas de sus miembros de forma fácil y/o completa ya que la potencial fuerza de trabajo disponible para que esto se diera no esta siendo ocupada; para los hogares pobres, el efecto es aún mayor, ya que viene a ser uno de los elementos que alargan -o perpetúan- su pobreza.

\section{Características socioeconómicas}

\subsection{Los beneficios recibidos por los hogares}

La investigación se centró principalmente en la medición de los beneficios producidos por las remesas en los hogares salvadoreños.

Aproximadamente, el $19 \%$ de los hogares salvadoreños tiene a alguno de sus miembros, por lo menos, residiendo en el exterior. Luego, el $23.2 \%$ de los hogares a que pertenecen los emigrantes, reciben ayuda en remesas. Esto significa que los migrantes ayudan ciertamente, al hogar al que pertenecen o pertenecieron, y también se dan migrantes que envían dinero a tíos, hermanos u otros parientes, y aún, amigos. En los últimos cuatro años, los departamentos con más hogares que poseen familiares en el extranjero y los que más ayuda reciben son: La Unión, Morazán, Cabañas, San Miguel y Chalatenango.

La remesa referida es en su mayoría, en dólares o divisas, la manera menos costosa de mandar ayuda. El $80 \%$ del total de la ayuda se 
recibe de la manera antes mencionada y el $2 \%$ de la remesa se recibe en especies. Los datos demuestran que durante los cuatro años, el porcentaje de hogares que recibió ayuda en efectivo, aumento, mientras que el porcentaje de hogares que recibió ayuda en especie, disminuyó; y los hogares que recibieron ayuda en efectivo y especies, también disminuyó. Esto significa que las personas que envían ayuda lo hacen en su mayoría en efectivo. Se presume que la ayuda en efectivo tiende a incrementar, como producto de los altos costos de enviar otro tipo de ayuda. Además, brinda mayor capacidad adquisitiva a los hogares receptores.

\section{Gráfico $N^{0} 7$}

Ayuda recibida 1999-2002

(En porcentajes)

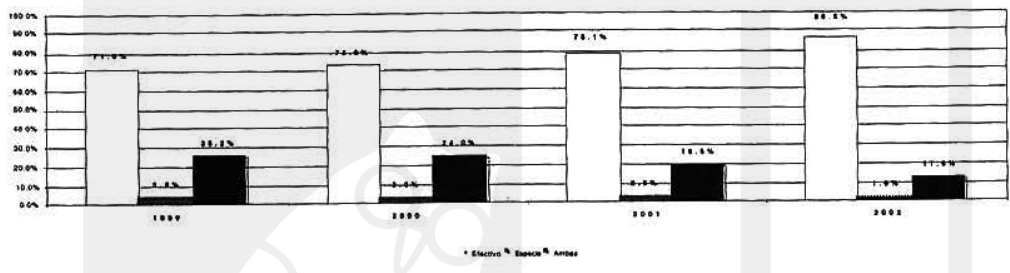

Fuente: Elaboración propia, sobre las bases de datos EHPM.

Promedio de hogares beneficiados

En promedio los hogares receptores de remesas de la zona urbana reciben $\$ 140$ mensuales; en la zona rural, el promedio es de $\$ 116$ mensuales. Nótese que es mayor el monto promedio recibido en la zona urbana. Es importante recalcar que el promedio recibido por hogar es casi el $98 \%$ del valor de la canasta básica, es decir, los hogares receptores de remesas, en su mayoría están situados encima de la línea de pobreza.

$\mathrm{Al}$ analizar la información más a fondo se encontró que $\mathrm{La}$ Unión es el departamento que recibe el mayor promedio de remesas y que el mayor porcentaje de hogares las reciben, tanto en lo urbano como en lo rural: $\$ 164$ y $\$ 145$, respectivamente. El $34 \%$ de los hogares urbanos en La Unión reciben remesas; y en la zona rural el $43.7 \%$ de los hogares. 


\section{Cuadro 2}

Montos promedio de ayuda recibida 1999-2002

(En Dólares)

\begin{tabular}{l|c|r|c|c|c|c|c|c}
\hline \multirow{2}{*}{ Departamentos } & \multicolumn{2}{|c|}{1999} & \multicolumn{2}{c|}{2000} & \multicolumn{2}{c|}{2001} & \multicolumn{2}{c}{2002} \\
\cline { 2 - 8 } & Urbana & Rural & Urbana & Rural & Urbana & Rural & Urbana & Rural \\
\hline Ahuachapán & 101 & 121 & 124 & 132 & 127 & 119 & 148 & 143 \\
Santa Ana & 131 & 96 & 107 & 109 & 152 & 136 & 149 & 127 \\
Sonsonate & 131 & 111 & 136 & 114 & 137 & 120 & 143 & 144 \\
Chalatenango & 95 & 87 & 112 & 118 & 144 & 107 & 147 & 136 \\
La Libertad & 139 & 98 & 128 & 89 & 133 & 124 & 182 & 144 \\
San Salvador & 122 & 51 & 128 & 104 & 158 & 117 & 158 & 126 \\
Cuscatlán & 136 & 68 & 143 & 110 & 158 & 177 & 190 & 137 \\
La Paz & 111 & 104 & 105 & 106 & 149 & 119 & 144 & 118 \\
Cabañas & 138 & 102 & 111 & 86 & 171 & 99 & 155 & 124 \\
San Vicente & 108 & 98 & 128 & 93 & 131 & 133 & 144 & 120 \\
Usulután & 123 & 98 & 143 & 111 & 152 & 102 & 172 & 139 \\
San Miguel & 117 & 99 & 131 & 107 & 165 & 107 & 172 & 116 \\
Morazán & 112 & 87 & 148 & 116 & 156 & 142 & 144 & 130 \\
La Unión & 143 & 128 & 155 & 130 & 154 & 152 & 200 & 168 \\
Promedio & 122 & 96 & 129 & 109 & 149 & 125 & 161 & 134 \\
\hline
\end{tabular}

Fuente: Elaboración propia, sobre las bases de datos EHPM.

\subsection{Remesas per cápita}

$\mathrm{Al}$ analizar el flujo de remesas junto con el estudio del ingreso per cápita mensual y el monto total de remesas por hogar, y distribuirlo en partes iguales entre el número de miembros que conforman los hogares salvadoreños, se obtuvieron los siguientes resultados:

- En El Salvador, las personas que forman parte de hogares receptores de remesas reciben mensualmente un promedio de $\$ 28.60$.

- La zona urbana presenta un mayor ingreso per cápita en concepto de remesas, alcanzando un monto de $\$ 33$, mientras que en el área rural fue de \$24.

- La Unión sigue siendo el departamento con mayor beneficio generado por las remesas, ya que éste refleja los montos recibidos por persona, con números más elevados tanto a nivel global (\$34) como en el área urbana (\$39) y rural (\$30). En segundo lugar, Cuscatlán 
con un promedio de remesa per cápita de $\$ 31$ y San Miguel con $\$ 30$.

- En el área urbana, además de La Unión, destacan San Miguel y Usulután, por tener los más altos indicadores per cápita de remesa recibida, con $\$ 36$ y $\$ 35$, respectivamente. En el área rural, Cuscatlán y Ahuachapán son los departamentos con los más elevados ingresos per cápita provenientes de remesas con $\$ 26$ y $\$ 25$ cada uno.

- En cada uno de los años que abarca la muestra, la remesa per cápita promedio ha ido en aumento. En el área rural el aumento ha sido del $45.2 \%$, en el área urbana el aumento registrado es menor, el cual fue de $37.1 \%$.

- En el área urbana, los departamentos que han registrado mayor crecimiento en el indicador per cápita, de recepción de remesas, en el periodo comprendido de 1999 a 2002, son Chalatenango (74.2\%) y Ahuachapán (55.0\%); por otro lado, Sonsonate $(9.4 \%)$ tiene la menor tasa de crecimiento.

- En el área rural, San Salvador y Cuscatlán, tuvieron el aumento más significativo de la remesa per cápita. Este es del $148.5 \%$ y $121.2 \%$, respectivamente. El menor se registro en San Miguel y fue del $21.2 \%$

\subsection{Destino de las remesas: una sociedad que apenas subsiste}

En cuanto al uso que los hogares receptores de remesas dan a las remesas, los datos demostraron que en su mayoría, son destinados al consumo: $90 \%$ en la zona rural y el $80 \%$ en la zona urbana. Tales porcentajes comprueban la hipótesis establecida en la investigación original, donde se sostiene que es el consumo el principal destino de las remesas familiares; sirviendo casi exclusivamente para la subsistencia de las personas. En los hogares receptores de remesas, el destino de las remesas al ahorro, es poco. Aún más: las remesas recibidas aumentaron, según reveló el estudio de los años investigados. No obstante, el ahorro en los hogares beneficiados no aumentó, y ni siquiera la inversión en cápital humano, y al contrario, aumentaron su consumo. 


\section{Cuadro 3}

Remesa per cápita promedio (dólares) y

tasa de crecimiento de remesa per cápita de 1999 a 2002

(En porcentajes)

\begin{tabular}{l|c|c|c|c|c}
\hline \multirow{2}{*}{ Departamento } & \multicolumn{5}{|c}{1999 a 2002 } \\
\cline { 2 - 5 } & Urbana & Rural & Total & Urbana & Rural \\
\hline Ahuachapán & 29 & 26 & 27 & $55.00 \%$ & $22.90 \%$ \\
Santa Ana & 34 & 24 & 29 & $22.30 \%$ & $37.20 \%$ \\
Sonsonate & 33 & 24 & 28 & $9.40 \%$ & $30.10 \%$ \\
Chalatenango & 29 & 23 & 26 & $74.20 \%$ & $58.50 \%$ \\
La Libertad & 36 & 24 & 30 & $36.10 \%$ & $55.80 \%$ \\
San Salvador & 34 & 21 & 28 & $32.80 \%$ & $148.50 \%$ \\
Cuscatlán & 35 & 26 & 31 & $44.00 \%$ & $121.20 \%$ \\
La Paz & 30 & 23 & 26 & $33.40 \%$ & $25.90 \%$ \\
Cabañas & 33 & 18 & 25 & $21.70 \%$ & $23.30 \%$ \\
San Vicente & 29 & 23 & 26 & $40.30 \%$ & $26.00 \%$ \\
Usulután & 36 & 25 & 30 & $43.80 \%$ & $51.90 \%$ \\
San Miguel & 36 & 24 & 30 & $49.50 \%$ & $21.20 \%$ \\
Morazán & 33 & 25 & 29 & $26.70 \%$ & $58.40 \%$ \\
La Unión & 39 & 30 & 34 & $47.30 \%$ & $35.40 \%$ \\
Total & 33 & 24 & 29 & $38.32 \%$ & $51.16 \%$ \\
\hline
\end{tabular}

Fuente: Elaboración propia, sobre las bases de datos EHPM.

Sin embargo, hay diferencias significativas: en la zona urbana, el $80 \%$ se destina aproximadamente al consumo mientras que en la zona rural es de $90 \%$ aproximadamente; esto es debido a que los hogares rurales cuentan con menores ingresos y por lo tanto se ven obligados a utilizar las remesas en su mayoría para cubrir gastos en necesidades básicas. Como la mayoría se destina al consumo, es realmente poco lo que les resta a los hogares para inversión, educación, salud y ahorro. En la zona urbana, el $3.5 \%$ es utilizado para inversión, $15 \%$ a inversión en cápital humano (salud y educación) y $2 \%$ en ahorro. En la zona rural, $2 \%$ en inversión, $8.5 \%$ en inversión en cápital humano y $1 \%$ en ahorro. En segundo lugar, después del consumo, los hogares en ambas zonas utilizan los fondos de remesas en educación y salud. A lo largo de estos años, se observó una disminución en la inversión 
en cápital humano tanto en la zona urbana como en la zona rural; en el 2001, se nota un incremento del uso de parte de las remesas, específicamente para vivienda, lo cual puede ser un resultado del terremoto de principios de año; luego indica que las remesas toman la figura de seguro ante desastres naturales o cualquier acontecimiento no previsto. En 2002, se vuelven a revertir los porcentajes, es decir, se aumenta de nuevo la inversión en cápital humano y disminuye la inversión. Durante estos años, el ahorro ha tendido a aumentar en la zona rural; el consumo ha disminuido en $1.1 \%$ y el ahorro aumentó en $0.2 \%$.

\subsection{Otras diferencias}

En El Salvador, al separar los hogares receptores de remesas de aquellos que no las reciben, se ve que en los primeros es mayor el porcentaje de mujeres como jefes de su hogar. Otra característica que se puede observar, es que en los hogares receptores de remesas el porcentaje de personas dentro de las edades de 65 años, es mayor que en los que no reciben: $11 \%$ para los receptores de remesas; $7 \%$ para los no receptores. Esta no es una afirmación tajante, pues, quizá, otra investigación social de mayor profundidad podría profundizar en ello.

Las cifras en ambas zonas indican que más de la mitad del país aún se encuentra en edades escolares (de 4 a 24 años de edad); esta alta proporción de personas jóvenes indica, por un lado, la necesidad de incrementar la inversión en cápital humano con el propósito de llegar a mejorar la calidad de vida de los habitantes; con respecto a las personas de la tercera edad, si bien representan un menor porcentaje comparados con el resto de la población, es necesario que sean atendidas y que se les garantice una existencia lo suficientemente digna. Ambas objetivos se ven obstaculizados por la baja productividad que el país presenta actualmente y la constante pobreza de la mayoría de la población, lo que resulta en casi nulas expectativas de mejoramiento de la calidad de vida del salvadoreño promedio.

\subsection{Déficit educativo simple de los individuos de HRR y HNRR zona urbana}

La información sobre el porcentaje de personas en edad de estudiar, que efectivamente estudian, indica que en la zona urbana no hay 
diferencia alguna entre los hogares receptores y no receptores, aunque los porcentajes promedio en los primeros experimentan una ligera alza.

Es oportuno señalar que los departamentos que presentan un porcentaje mayor en la zona urbana de los HRR son La Libertad (70.0\%) y Usulután (68.7\%). Asimismo, los que destacan más en los HNRR, son La Libertad (60.9\%) y San Salvador (59.2\%). De los HRR urbanos con porcentaje menor, destacan Ahuachapán (54.1\%) y La Unión $(59.4 \%)$. En los HNRR, los que presentan los porcentajes más pequeños en relación a los otros, son La Unión (45.6\%) y Ahuachapán (46.7\%). Al analizar esta información, se observa que La Unión y Ahuachapán, independientemente de que obtengan remesas o no, son los que tienen menores porcentajes de personas que reciben educación escolar en relación a los demás departamentos.

\section{Cuadro $N^{\circ} 10.1$}

Porcentaje de Estudiantes por hogar/ Personas en edad de estudiar (de 4 a 24 años)

De los HRR y HNRR: Zona Urbana

\begin{tabular}{l|c|c|c|c|c}
\hline Hogares & 1999 & 2000 & 2001 & 2002 & Promedio \\
\hline Receptores & 64.4 & 65.7 & 56.4 & 56.0 & 60.6 \\
No Receptores & 60.4 & 60.1 & 60.0 & 62.1 & 60.7 \\
\hline
\end{tabular}

Fuente: Elaboración propia, sobre las bases de datos EHPM.

\subsection{Zona rural}

En la zona rural, los resultados son distintos: si bien en la zona urbana el porcentaje promedio de individuos que estudian no varía, en la zona rural hay variación, pues es mayor en $11 \%$ en los hogares receptores. El dato de remesas indica que en la zona rural, los hogares que reciben remesas tienen mayor acceso a educación que los que no reciben remesas. 


\section{Cuadro $\mathrm{N}^{\circ} 10.2$}

Porcentaje de estudiantes por hogar/ personas en edad de estudiar (de 4 a 24 años)

De los HRR y HNRR: Zona Rural

\begin{tabular}{l|c|c|c|c|c}
\hline Hogares & 1999 & 2000 & 2001 & 2002 & Promedio \\
\hline Receptores & 53.9 & 57.7 & 56.4 & 56.0 & 56.0 \\
No Receptores & 42.4 & 45.3 & 45.1 & 47.1 & 45.0 \\
\hline
\end{tabular}

Fuente: Elaboración propia, sobre las bases de datos EHPM.

Es claro que a lo largo de los cuatro años aumentó el porcentaje de individuos que efectivamente aprovechan la educación, tanto en los HRR como en los HNRR, lo que implica un leve avance de la educación rural.

La información indica que en la zona rural, los departamento que presentan mayor porcentaje de estudiantes con respecto al total en edad de estudiar en los HRR, son Cuscatlán (68.0\%); San Miguel (59.5\%); y La Paz (58.6\%). Por otro lado, en los HNRR sobresalen Cuscatlán (51.2\%); Chalatenango (50.9\%); y San Salvador $(50.2 \%)$. Se deduce que Cuscatlán es, a nivel rural, el departamento con mayor porcentaje de personas que se están educando, ya sea si reciben remesas o no.

Luego, los que tienen menor proporción de personas que estudian con respecto al total en edad de estudiar son: en los HRR, La Unión (47.6\%); Ahuachapán (51.3\%); y Cabañas (51.4\%); y en los HNRR, La Unión (37.0\%); Ahuachapán (39.5\%); y Santa Ana (39.8\%). Dando como resultado que La Unión y Ahuachapán son los que tienen menos personas asistiendo a centros educativos.

Es claro como independientemente de la zona, ya sea urbana y rural; y del tipo de hogar, ya sea receptor de remesas o no, La Unión y Ahuachapán son los departamentos salvadoreños que se llevan la peor parte en lo referente a la educación.

\subsection{Hallazgos importantes}

Si las remesas no existieran o, mejor dicho, si nunca se hubiese presentado el fenómeno migratorio estudiado, habría aumentado la 
pobreza o surgido la miseria en esos hogares pobres, amén de que la brecha de la pobreza se hubiese ensanchado. Específicamente, las remesas aumentaron el ingreso familiar en la zona urbana en un $8.2 \%$; y en la zona rural en $9.7 \%$.

Al no haber existido las remesas, la pobreza en estos hogares pobres, se hubiese aumentado, además, de haber surgido otros hogares pobres. Este fenómeno creciente de pobreza, y quizá de miseria, aumentaría en un $13.2 \%$ en la zona urbana, y un $11.2 \%$ en la zona rural. Las remesas acortan la brecha de la pobreza en un $4.9 \%$ en la zona urbana; y en $6.2 \%$ en la zona rural.

\section{El Salvador sin remesas: ¿Cuánto bajaría el ingreso?}

\subsection{Ingreso familiar sin remesas}

Si no existieran remesas en el país, el ingreso familiar de la población salvadoreña se reduciría en promedio a lo largo de los cuatro años en un $8.7 \%$; los resultados se pueden observar a continuación, para los cuatro años, tanto a nivel nacional, como para la zona urbana y la rural:

\section{Grafico $\mathrm{N}^{\circ} 11$}

Ingreso familiar promedio con y sin remesas 1999- 2002

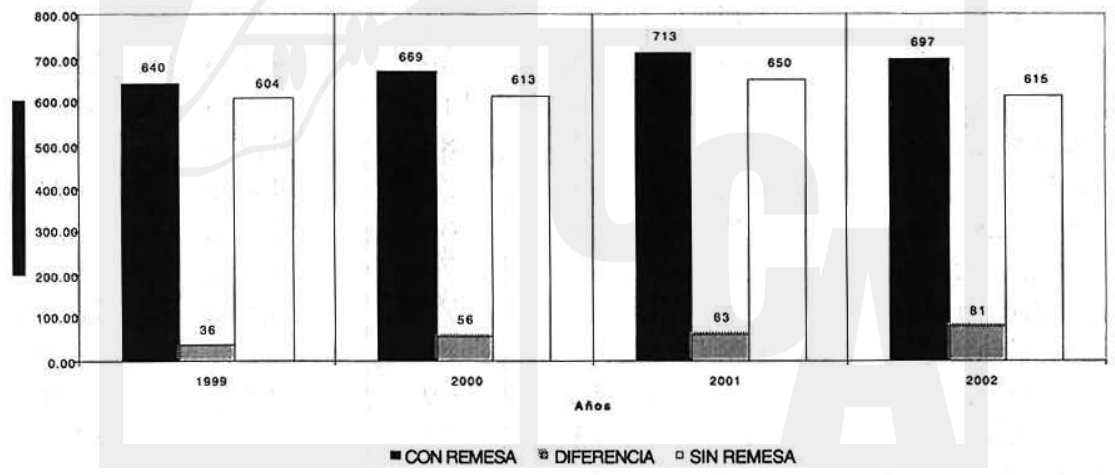

Fuente: Elaboración propia, sobre las bases de datos EHPM. 
De no haber remesas, el poder adquisitivo de la población total se vería afectado; y es de destacar que la zona rural es la más perjudicada, ya que es en ésta donde hay una mayor dificultad de acceso a los servicios básicos y otros elementos - vías de acceso, oportunidad de empleo, etc.- que puedan mejorar la calidad de vida de los habitantes. Si bien el ingreso familiar promedio sin remesa, ha tendido a aumentar, aún no es suficiente como para que las remesas dejen de ser un porcentaje importante. Lo que demuestra a su vez, que las remesas son las que sostienen en gran parte la economía familiar salvadoreña.

\subsection{Ingreso per cápita excluyendo remesas}

Al analizar el ingreso per cápita en El Salvador, excluyendo el ingreso proveniente de las remesas, nos encontramos con los siguientes resultados:

\section{Cuadro $\mathrm{N}^{\circ} 5$}

Ingreso promedio per cápita, excluyendo remesas 1999 a 2002 (En dólares)

\begin{tabular}{l|c|c|c|c|c}
\hline \multirow{2}{*}{ Departamento } & \multicolumn{3}{|c|}{ Promedio } & Tasa Crec & Tasa Crec. \\
\cline { 2 - 6 } & Urbano & Rural & País & Urbano & Rural \\
\hline Ahuachapán & 91 & 43 & 67 & $-5.0 \%$ & $4.3 \%$ \\
Santa Ana & 111 & 47 & 79 & $11.8 \%$ & $14.7 \%$ \\
Sonsonate & 119 & 47 & 83 & $9.4 \%$ & $7.7 \%$ \\
Chalatenango & 107 & 40 & 73 & $2.3 \%$ & $-3.9 \%$ \\
La Libertad & 204 & 65 & 134 & $4.3 \%$ & $14.5 \%$ \\
San Salvador & 162 & 61 & 111 & $11.8 \%$ & $16.5 \%$ \\
Cuscatlán & 107 & 64 & 85 & $-4.4 \%$ & $1.0 \%$ \\
La Paz & 102 & 51 & 77 & $4.6 \%$ & $14.0 \%$ \\
Cabañas & 98 & 28 & 63 & $5.4 \%$ & $3.7 \%$ \\
San Vicente & 106 & 37 & 71 & $3.9 \%$ & $5.4 \%$ \\
Usulután & 113 & 41 & 77 & $15.6 \%$ & $16.4 \%$ \\
San Miguel & 118 & 40 & 79 & $-1.0 \%$ & $3.2 \%$ \\
Morazán & 102 & 36 & 69 & $19.7 \%$ & $15.9 \%$ \\
La Unión & 100 & 43 & 72 & $3.2 \%$ & $14.9 \%$ \\
Total Promedio & 117 & 46 & 82 & $5.8 \%$ & $9.2 \%$ \\
\hline
\end{tabular}

Fuente: Elaboración propia, sobre las bases de datos EHPM. 
- El ingreso per cápita promedio a nivel nacional es de $\$ 82$ al mes. En el área urbana, el ingreso por persona es de $\$ 117$; y en el área rural es de $\$ 46$ el ingreso promedio.

- El departamento que muestra el ingreso per cápita más alto es La Libertad, con un ingreso promedio de \$134; \$204 en el área urbana y $\$ 65$ en el área rural. San Salvador es después de La Libertad, el departamento con mayores ingresos, a nivel nacional, con un promedio per cápita promedio de $\$ 111$; y de $\$ 162$ en el área urbana. Cuscatlán con $\$ 64$ de ingreso por persona, es el departamento que presenta los ingresos más elevados luego de La Libertad, en la zona rural.

- Los ingresos per cápita más bajos se presentan en los departamentos de Cabañas, Ahuachapán y Morazán, con cantidades de \$63, $\$ 67$ y $\$ 69$ respectivamente. En el área urbana, Ahuachapán (\$91), Cabañas (\$98) y La Unión (\$100) presentan los promedios más bajos de ingresos. En el área rural es de nuevo Cabañas con $\$ 28, y$ se agregan Morazán (\$36) y San Vicente (\$37) como los departamentos con los menores ingresos por persona.

- En el área rural, se experimentó un crecimiento de $9.2 \%$ del ingreso per cápita entre los años 1999 y 2002; esta tasa es mayor a la que se obtuvo en el área urbana la cual fue de $5.8 \%$. Morazán y Usulután destacan como los departamentos con mayores tasas de crecimiento del ingreso por persona en el área urbana, ya que dicha tasa fue de $19.7 \%$ y $15.6 \%$ respectivamente; también es de mencionar que los departamentos de Ahuachapán, Cuscatlán y San Miguel experimentaron reducciones en sus ingresos per cápita. De los hogares provenientes de la zona rural destacan los originarios de San Salvador y Usulután con tasas de crecimiento del $16.5 \%$ y $16.4 \%$ respectivamente; y el departamento de Chalatenango cuyo ingreso per cápita se redujo en un $3.9 \%$.

El orden de los departamentos más y menos pobres no varía en su ingreso per cápita aún si se restan las remesas.

\subsection{Impacto de las remesas en concepto del ingreso per cápita}

El ingreso per cápita se reduce en un $9.7 \%$ en promedio, cuando no se incluye el ingreso por remesas; la reducción es mayor en el área rural con $13.3 \%$, y es de $8.2 \%$ la reducción en el área urbana. 
Los departamentos que sufren una disminución más significativa en el ingreso per cápita, cuando no se incluye el ingreso en concepto de remesas, son La Unión, Morazán y Cabañas, con una reducción del $18.5 \%, 14.0 \%$ y $13.4 \%$, respectivamente. Por el contrario, en San Salvador y La Libertad la reducción en el ingreso per cápita, cuando no son incluidas las remesas, es menor que el $5 \%$.

La Unión tiene mayores ingresos por remesas en proporción al ingreso total; después Cabañas y Morazán destacan como los departamentos que registran mayor sensibilidad en el ingreso per cápita ante la reducción total de las remesas. Simplificando: de no tener remesa alguna, La Unión, Cabañas y Morazán serían los departamentos donde el ingreso promedio por persona bajaría más, afectando el bienestar de sus habitantes.

\section{Cuadro $\mathrm{N}^{\circ} 6$}

Reducción del ingreso per cápita, excluyendo remesas

(En porcentajes)

\begin{tabular}{|l|r|r|r|}
\hline \multirow{2}{*}{ Departamento } & \multicolumn{3}{|c|}{ Promedio } \\
\cline { 2 - 4 } & Urbano & Rural & \multicolumn{1}{|c|}{ País } \\
\hline Ahuachapán & $-6.7 \%$ & $-10.0 \%$ & $-7.8 \%$ \\
Santa Ana & $-8.6 \%$ & $-13.4 \%$ & $-10.1 \%$ \\
Sonsonate & $-6.5 \%$ & $-5.6 \%$ & $-6.2 \%$ \\
Chalatenango & $-8.7 \%$ & $-15.9 \%$ & $-10.8 \%$ \\
La Libertad & $-3.8 \%$ & $-6.1 \%$ & $-4.3 \%$ \\
San Salvador & $-3.7 \%$ & $-5.2 \%$ & $-4.1 \%$ \\
Cuscatlán & $-9.0 \%$ & $-4.7 \%$ & $-7.4 \%$ \\
La Paz & $-8.5 \%$ & $-9.3 \%$ & $-8.7 \%$ \\
Cabañas & $-11.3 \%$ & $-20.0 \%$ & $-13.4 \%$ \\
San Vicente & $-6.3 \%$ & $-10.8 \%$ & $-7.5 \%$ \\
Usulután & $-8.6 \%$ & $-15.3 \%$ & $-10.5 \%$ \\
San Miguel & $-10.3 \%$ & $-17.4 \%$ & $-12.2 \%$ \\
Morazán & $-9.6 \%$ & $-24.6 \%$ & $-14.0 \%$ \\
La Unión & $-13.9 \%$ & $-27.4 \%$ & $-18.5 \%$ \\
Total Promedio & $-8.2 \%$ & $-13.3 \%$ & $-9.7 \%$ \\
\hline
\end{tabular}

Fuente: Elaboración propia, sobre las bases de datos EHPM. 


\section{Los pobres de El Salvador sin remesas}

Sin remesas, la estabilidad macroeconómica que se ha logrado no hubiera sido posible. La investigación proporcionó una cuantificación del incremento de la pobreza en El Salvador, al comparar los resultados de los hogares que se encuentran en pobreza extrema y relativa, con el caso hipotético de que estos mismos hogares no recibiesen remesa alguna: se determinó en cuánto se reduciría el ingreso familiar mensual y en cuánto aumentarían los porcentajes de hogares pobres, así como el incremento de la profundidad de la pobreza y en que departamentos habría mayor o menor profundidad; además, se indica en cuanto aumentará el factor que al multiplicarlo por los ingresos, cierra la brecha de pobreza. De esta manera, se conoce si los departamentos actualmente más pobres siguen en ésta misma situación o sí, por el contrario surgen otros departamentos con mayores grados de pobreza.

\subsection{Pobreza extrema sin remesas}

Si El Salvador no recibiera remesas, la composición de la pobreza sería aún más alarmante. Al analizar el ingreso familiar por zona, se deduce lo siguiente: en la zona urbana la pobreza, durante los años estudiados, hubiese aumentado en $19.25 \%$. Si no se hubieran recibido remesas, los departamentos en cuyos hogares hubieran disminuido más los ingresos, serían San Miguel y La Unión mientras que San Vicente y $\mathrm{La} \mathrm{Paz}$ tendrían reducciones relativamente pequeñas, haciéndolos los departamentos que menores reducciones sufrirían.

A lo largo de los cuatro años estudiados, el ingreso familiar disminuiría aproximadamente en un $13.1 \%$. Luego, los departamentos posiblemente más afectados son La Unión con un $27 \%$ y Morazán con un $17.1 \%$; y los relativamente menos afectados serían los departamentos de San Salvador, con 5\%, y Ahuachapán con $6.9 \%$. 


\section{Cuadro 7}

Porcentajes de reducción del ingreso promedio de los hogares pobres de no existir remesas.

Zonas urbana y rural

\begin{tabular}{|l|r|r|}
\hline Departamento & Urbano & Rural \\
\hline Ahuachapán & $17.70 \%$ & $6.80 \%$ \\
Santa Ana & $18.50 \%$ & $11.70 \%$ \\
Sonsonate & $16.80 \%$ & $13.20 \%$ \\
Chalatenango & $21.80 \%$ & $12.40 \%$ \\
La Libertad & $19.70 \%$ & $8.90 \%$ \\
San Salvador & $19.40 \%$ & $5.00 \%$ \\
Cuscatlán & $24.40 \%$ & $12.50 \%$ \\
La Paz & $13.00 \%$ & $12.20 \%$ \\
Cabañas & $20.40 \%$ & $15.20 \%$ \\
San Vicente & $9.20 \%$ & $8.50 \%$ \\
Usulután & $18.90 \%$ & $14.20 \%$ \\
San Miguel & $22.10 \%$ & $15.80 \%$ \\
Morazán & $19.90 \%$ & $17.10 \%$ \\
La Unión & $26.20 \%$ & $27.00 \%$ \\
Promedio & $19.14 \%$ & $12.89 \%$ \\
\hline
\end{tabular}

Fuente: Elaboración propia, sobre las bases de datos EHPM.

El porcentaje de hogares en pobreza extrema, de no recibir divisas, se incrementaría en un $5.9 \%$ en la zona urbana en los cuatro años; y el porcentaje de pobreza extrema en los hogares rurales, aumentaría en un $8.48 \%$. En cuanto al aumento de la profundidad de la pobreza, en promedio, un hogar urbano que se encuentre en la pobreza extrema, tendría que ganar $\$ 15.13$ ( $\$ 190.03)$ más para poder salir de tal situación; en la zona rural, la cantidad sería de $\$ 6.59$ ( $\$ 57.66)$. Cantidades difíciles de alcanzar, dada la mala situación económica en que están hundidos.

Con respecto al factor por el que se debe multiplicar el ingreso para comprobar, con el resultado, la cantidad necesaria para que estos hogares puedan salir de la pobreza, media vez les ingresara. Este factor experimenta un marcado aumento: en la zona urbana, en promedio el ingreso se tendría que multiplicar por 0.71 ; si no hubiera remesas, 
este número aumentaría a 1.17; es decir, el ingreso debería de multiplicarse 0.46 veces más; la zona rural tiene un caso análogo; de no tener remesas, los hogares rurales pasarían de multiplicar su ingreso por 0.94 a 1.24 para salir de la pobreza extrema, esto equivale a un aumento de 0.30 en dicho factor.

\subsection{Pobreza relativa sin remesas}

$\mathrm{Al}$ igual que con la pobreza extrema, la pobreza relativa en El Salvador aumentaría en grado sumo, al no recibir remesas: los montos de ingreso familiar mensual se reducirían en $13.2 \%$ en la zona urbana. En La Unión y Chalatenango, de no recibir remesas, el número de hogares en pobreza relativa aumentaría, pues sus ingresos familiares disminuirían más que en los otros departamentos. En el otro extremo, la pobreza relativa aumentaría relativamente menos en San Salvador y San Vicente. Esto es, el ingreso familiar en estos dos departamentos se reduciría en porcentajes menores que los del resto citado.

\section{Cuadro $\mathrm{N}^{\circ} 7$}

Reducción del ingreso damiliar promedio de los hogares

en pobreza eelativa

sin remesas: zonas urbana y rural

(En porcentajes)

\begin{tabular}{|l|r|r|}
\hline Departamento & Urbano & Rural \\
\hline Ahuachapán & $12.80 \%$ & $5.50 \%$ \\
Santa Ana & $13.80 \%$ & $11.30 \%$ \\
Sonsonate & $9.60 \%$ & $4.00 \%$ \\
Chalatenango & $18.50 \%$ & $15.90 \%$ \\
La Libertad & $11.10 \%$ & $6.60 \%$ \\
San Salvador & $9.00 \%$ & $5.40 \%$ \\
Cuscatlán & $12.00 \%$ & $5.80 \%$ \\
La Paz & $10.10 \%$ & $7.50 \%$ \\
Cabañas & $18.20 \%$ & $16.20 \%$ \\
San Vicente & $9.30 \%$ & $8.10 \%$ \\
Usulután & $13.50 \%$ & $12.50 \%$ \\
San Miguel & $15.20 \%$ & $13.50 \%$ \\
Morazán & $10.70 \%$ & $18.80 \%$ \\
La Unión & $20.70 \%$ & $25.20 \%$ \\
Total & $13.20 \%$ & $11.23 \%$ \\
\hline
\end{tabular}

Fuente: Elaboración propia, sobre las bases de datos EHPM. 
En la zona rural, el ingreso se reduciría en un $11.2 \%$ a lo largo del período de cuatro años. Se ve que en la zona urbana, resaltan los mismos departamentos, principalmente La Unión (25.2\%); los relativamente menos afectados resultarían ser Sonsonate (4\%), y San Salvador $(5.5 \%)$.

Los promedios del porcentaje de hogares en pobreza relativa, aumentarían en la zona urbana en $4.9 \%$; y en la rural, en $6.2 \%$.

En la zona urbana, la profundidad de la pobreza relativa aumentaría a niveles aún más impresionantes si no se recibieran remesas: un promedio de $13.7 \%$ en el período estudiado. En la zona rural también se presentarían aumentos de la profundidad en la pobreza relativa, un $9.3 \%$ en promedio.

El factor que se tendría que multiplicar por el ingreso familiar mensual, dará el porcentaje adicional del ingreso, para superar la pobreza relativa, obtenido este porcentaje se observa que urge un claro aumento en ambas zonas del país: a nivel urbano es de 0.82; de no recibirse ayuda alguna, el factor se incrementaría en 0.28 , lo que aumentaría a 1.10, en la zona rural. Coincidentemente, habría pues, un incremento análogo, llevando el factor de 1.10 a 1.38 .

De la comparación entre la situación real de El Salvador de su pobreza relativa y extrema, con la situación hipotética ensayada (no recibir remesas), resulta que las remesas son uno de las aportaciones principales para mantener a flote la economía familiar, sirviendo de alivio -más no de cura - a la pobreza de ingresos. Sería mucho mayor la pobreza al no recibir tales transferencias; asimismo, se observa cómo la política social del gobierno salvadoreño de los últimos años, no ha logrado iniciar una reducción de la pobreza, ya que si bien es cierto que los ingresos han aumentado, la recepción de remesas ha sido uno de los elementos que ha permitido dicho crecimiento.

\subsection{Tasa media de dependencia de los HRR vs. HNRR}

La investigación mostró que los hogares con ayuda en concepto de remesas, tienen tasas de dependencia mayores que aquellos hogares sin esa ayuda.

Los HRR muestran en los años de 1999 a 2002, una tasa de dependencia promedio de 2.2 por hogar; 2.0 en promedio en el área urbana y 2.3 en promedio para el área rural. 
Los departamentos que tienen mayor proporción de miembros dependientes por hogar (desocupados en relación a ocupados) son: $\mathrm{La}$ Unión, Cabañas, San Vicente y Ahuachapán. En la zona urbana la tasa de dependencia promedio es menor que en la zona rural.

La relación entre los HRR y la tasa de dependencia promedio, demuestra que los departamentos con proporción de HRR más elevada, poseen las tasas de dependencia más altas. Lo anterior se observa en La Unión y Cabañas. Por el contrario, los departamentos que presentan menor proporción de HRR poseen tasas de dependencia menores; este puede ser el caso de La Libertad y San Salvador. Como se expuso con anterioridad, los HNRR muestran tasas de dependencia menores que los HRR, por el hecho de que los hogares sin ese ingreso extra proveniente del exterior, se ven obligados de manera más urgente, a suplir sus necesidades buscando empleo insistentemente y así ocupan mayor número de miembros del hogar.

La tasa media de dependencia en los HNRR durante los cuatro años de esta investigación, es de 1.9 por hogar. En el área urbana este indicador asciende a 1.7, y en el área rural es de 2.2 miembros desocupados por miembro ocupado. Estas tasas son de 0.3 miembros menos que la que registran los HRR tanto a nivel nacional como en el área urbana, mientras, en el área rural es tan sólo 0.1 miembros menor.

El departamento de Cabañas sobresale, por ser el que posee la más alta tasa de dependencia tanto a nivel nacional, como en el área urbana como en la rural. Ahuachapán es el segundo en este aspecto. Si hacemos una comparación entre el departamento que posee las tasas de dependencia más elevadas tanto para los HRR como para los HNRR, observamos que la tasa de dependencia es menor para los HRR, así: a nivel general en 0.1 miembros por hogar, para la zona urbana la tasa de dependencia es menor en 0.4 , y los hogares rurales es mayor en 0.1 miembros.

Al observar cada año, el promedio total en las zonas urbana y rural, resalta el hecho de que las tasas de dependencia son en todos los casos, mayores en los HRR, principalmente en el área urbana. En conclusión, los hogares receptores de remesa (HRR) poseen tasas de dependencia más elevadas en comparación a las registradas por los hogares que no reciben tal ayuda. Sin embargo, la diferencia observada no es de gran magnitud, principalmente, en los hogares pertenecientes al área rural. 


\section{Conclusiones y reflexiones}

\subsection{Causas del aumento de remesas}

Se detectaron en la investigación dos causas del aumento de remesas. Primero, ya se dijo, los emigrantes buscan mejores condiciones de vida y más que todo, para sus familias de las que se alejan temporalmente o con la idea de, una vez instalados en el exterior, llevar a su cónyuge e hijos a su nueva residencia. Segundo, los ingresos de las personas domiciliadas en otros países, aumentan.

\subsection{Desaceleración y desempleo}

Estados Unidos, país en donde se encuentra una enorme cantidad de salvadoreños, sufre desde 2001 una desaceleración de su economía ${ }^{6}$, lo que se traduce en aumentos en la tasa de desempleo. Esta situación dio lugar a pensar, que las remesas disminuirían considerablemente. Sin embargo, el pronóstico no se cumplio, y, todo lo contrario, las remesas aumentaron en cantidad y en número de hogares favorecidos. Estudios de terceros corroboran nuestras afirmaciones, ya que "en los Estados Unidos hay una aparente demanda estructural por los trabajadores extranjeros (...) que parece estar a prueba de recesiones, incluso después de los ataques del 11 de septiembre de 2001"?

Se concluye con ello que las remesas no se ven afectadas por los determinantes de los flujos de cápital, en mejores palabras, como la mayoría de empleos de los salvadoreños en aquel país se encuentran en los servicios - limpieza de casas, edificios, cuido de niños y familiares- el decrecimiento de la economía estadounidense no impactó el envío de remesas.

\subsection{La mejoría producida por las remesas y las políticas sociales}

Los emigrantes salvadoreños buscan ascender en lo económico, social y cultural; y, sobretodo, obtener mejores salarios por su trabajo, que los que podrían alcanzar en su patria, así como enviar dólares a sus familias. Tal panorama se destaca precisamente por el estancamiento de los sectores víctimas de la pobreza. El impacto positivo de las remesas en la familia urbana y rural salvadoreña no debe ser la base de nuestro desarrollo. Más bien, se estima que urgen nuevas políticas económicas que fomenten la productividad de los hogares.

\section{4}

Realldad 97, 2004 
Es necesario un fortalecimiento institucional interno, y una política económica que enfatice más lo microeconómico y el aspecto social. La migración es imparable. Incluso, de haber cambios estructurales en las economías, la gente podría seguir emigrando: en este país ya no hay lugar suficiente para todos. La sobrepoblación es un hecho que difícilmente se pueda cambiar. ¿Será la migración algo que ya se arraigó en los salvadoreños?

La verdadera búsqueda de igualdad socioeconómica es algo difícil de iniciar, ya que el poder económico está siempre vinculado al poder político (sino, véase la realidad salvadoreña: donde miembros prominentes de importantes conglomerados empresariales han sido parte de la cúpula del partido gobernante) y este poder político mal implementado puede ser, más que un facilitador, un impedimento para el alcance de la equidad. En el ámbito internacional es lo mismo: sino, mírese como los países del primer mundo erigen barreras a la inmigración y a los productos agrícolas - algo que no están dispuestos a ceder: véase las dificultades en las negociaciones de los sectores agrícolas centroamericanos con su contraparte estadounidense en el contexto del TLC_, lo que a la larga agranda las brechas entre Tercer y Primer Mundo.

\section{Bibliografía}

- Revista Business Week.

- Castro, A; Parada, C; Guevara, F; Carbajal, M. "Impacto de las Remesas Familiares en la Economía de los Hogares Salvadoreños", Trabajo de graduación para optar al grado de Licenciado en Economía, UCA, Septiembre de 2003.

- Periódico Financial Time.

- Periódico La Prensa Gráfica.

- Lagace, Martha, "What World Migration Means for Business", HBS Working Knowledge Newsletter www.hbswk.hbs.edu

- Scott, Bruce R. "Great Divide in the Global Village" HBS Working Knowledge Newsletter http://hbswk.hbs.edu 


\section{Notas}

1. Scott, Bruce R. "Great Divide in the Global Village" HBS Working Knowledge Newsletter http://hbswk.hbs.edu

2. Trabajo de graduación para optar al grado de Licenciado en Economía, por Álvaro Castro, Claudia Parada, Francisco Guevara y María Reneé Carbajal. Septiembre de 2003.

3. El Banco Mundial hace una clasificación del ingreso para propósitos operacionales y analíticos, basado en el concepto de ingreso nacional bruto per cápita y, en el INB per cápita y en el método Atlas del Banco Mundial. Los paises pueden ser clasificados en las siguiente categorías: ingreso bajo, US \$735 o menos; ingreso medio bajo, $\$ 736$ a $\$ 2935$; ingreso medio alto, $\$ 2936$ a $\$ 9075$; e ingreso alto, $\$ 9076$ o más. Las tablas del Banco Mundial clasifican a todos los países miembros y no miembros (para un total de 208) que tengan poblaciones mayores de 30,000 personas. Se hacen además clasificaciones adicionales en regiones geográficas y niveles de deuda externa. La clasificacion hecha por ingreso no refleja necesariamente el estado de desarrollo de los países.

4. La línea de pobreza para cada año es discinta, ya que esta es determinada por el valor de la Canasta Básica Alimenticia (CBA), conformada a partir del Índice de Precios al Consumidor (IPC), creado en 1992.

5. Pobreza relativa se refiere a aquellas personas u hogares cuyos ingresos son mayores que el costo de la CBA, pero son menores que el costo de la $\mathrm{CA}$, el cual, de acuerdo con la metodología del Banco Mundial, resulta ser el doble del costo de la CBA.

6. La economía estadounidense ha empezado a mostrar signos de una segura pero muy lenta recuperación a partir de los últimos meses de 2003. www.ft.com, www.businessweek.com, www.laprensagrafica.com.sv.

7. Lagace, Martha, "What World Migration Means for Business", HBS Working Knowledge Newsletter www.hbswk.hbs.edu 